\title{
Designing a Vehicle Mounted High Resolution Multi-Spectral 3D Scanner - Concept Design
}

\author{
Gregory Meyers \\ University of Sheffield \\ g.m.meyers@sheffield.ac.uk \\ Danielle Densley Tingley \\ University of Sheffield \\ d.densleytingley@sheffield.ac.uk
}

\author{
Chengxi Zhu \\ University of Sheffield \\ czhu10@sheffield.ac.uk \\ Jon Willmott \\ University of Sheffield \\ j.r.willmott@sheffield.ac.uk
}

\author{
Martin Mayfield \\ University of Sheffield \\ martin.mayfield@sheffield.ac.uk \\ Daniel Coca \\ University of Sheffield \\ d.coca@sheffield.ac.uk
}

\begin{abstract}
Buildings can improve their energy efficiency through retrofitting and thus decrease energy demand throughout the life of the building. However, evaluating building retrofit opportunities at a city level is a significant challenge. This requires identifying where in the city the biggest energy efficiency gains can be made and in the most costeffective way. A surveyor is typically relied upon to manually assess a building for insulation absence, defective installation, thermal leakage and other similar issues. To perform these inspections across whole cities would be prohibitively time intensive. There is therefore a need for a faster approach to detect and prioritise a city's retrofit requirements so that effective value for money decisions can be made. In this paper, the concept design of a vehicle mounted integrated sensing platform to collect high resolution visual, thermal and 3D scene data of the built environment at a city scale is presented. Initial design considerations are first explored before an initial concept design is presented and evaluated. From the evaluation, a number of concerns about the design were raised. Based on these findings, a significantly revised concept design is subsequently presented that addresses the aforementioned issues.
\end{abstract}

\section{CCS CONCEPTS}

- Hardware $\rightarrow$ Analysis and design of emerging devices and systems; Sensor devices and platforms; • Human-centered computing $\rightarrow$ Ubiquitous and mobile computing design and evaluation methods.

\section{KEYWORDS}

system design, sensors, mobile mapping, thermography, LiDAR

\section{ACM Reference Format:}

Gregory Meyers, Chengxi Zhu, Martin Mayfield, Danielle Densley Tingley, Jon Willmott, and Daniel Coca. 2019. Designing a Vehicle Mounted High Resolution Multi-Spectral 3D Scanner - Concept Design. In The 2nd Workshop on Data Acquisition To Analysis (DATA'19), November 10, 2019, New York, NY, USA. ACM, New York, NY, USA, 6 pages. https://doi.org/10.1145/ 3359427.3361921

Permission to make digital or hard copies of part or all of this work for personal or classroom use is granted without fee provided that copies are not made or distributed for profit or commercial advantage and that copies bear this notice and the full citation on the first page. Copyrights for third-party components of this work must be honored

For all other uses, contact the owner/author(s).

DATA'19, November 10, 2019, New York, NY, USA

(C) 2019 Copyright held by the owner/author(s).

ACM ISBN 978-1-4503-6993-0/19/11.

https://doi.org/10.1145/3359427.3361921

\section{INTRODUCTION}

The continuous increase of global population and consumption of natural resources requires the development of cities to develop ecologically $[2,17]$. The concept of an eco-city is to create sustainable urban systems considering aspects of the land usage, environment protection, closed-loop life support systems and urban life quality [10]. The development of life support systems, such as water, energy and waste management, are fundamental elements to building a sustainable city. For efficient urban energy management, a city level prioritisation of retrofit is essential. Through retrofitting, buildings can improve their energy efficiency and thus decrease energy demand throughout the life of the building, this is also often substantially more cost-effective and viable than a completely new construction. However, making building retrofit a priority at the city level is a significant challenge as this requires an understanding of power consumption, thermal distribution and construction conditions over the whole urban area and across different seasons $[5,6,15]$.

At present, the majority of approaches for the detection of insulation absence, defective installation, thermal leakage, presence of moisture and other similar issues rely on qualitative examination of captured thermographs for visual identification of differing temperature profiles by a surveyor [7]. To conduct this at scale across a city would be prohibitively time intensive. There is therefore a need for a faster approach to detect and prioritise a city's retrofit requirements.

Through the advancement and application of Computer Vision (CV) and Machine Learning (ML) methods on captured urban scene data, significant advances have been made in many areas such as built infrastructure monitoring, autonomous driving and urban planning. However, even with recent advancements there are currently many tasks that still remain out of reach. For some of these tasks, the primary issue lies not in the insufficiency of the available $\mathrm{CV}$ and ML methods, but with the more fundamental issue of having insufficient data for these methods to be properly utilised. Thus while many urban scene datasets exist, most are limited in not only scene scale, resolution, completeness and variation, but also most importantly in sensor data diversity.

Urban 3D modelling has been studied over several decades, accompanied with the emergence of many novel systems to achieve this purpose $[12,14]$. These systems are usually equipped on low flying aircraft or Unmanned Aerial Vehicles (UAVs) flying over the city or on vehicles driving through the city to collect the data $[4,9]$. LiDAR systems have been used to build 3D urban maps by 
generating large amounts of cloud point data that is processed by reconstruction algorithms $[3,11]$. Through combining scene images and cloud point data, highly accurate 3D urban maps can be developed with greater detail $[16,18]$. To obtain the external thermal distribution of buildings, it is also necessary to capture and combine co-registered infrared information $[1,8,13,15,19]$.

In this paper, the initial concept design of a vehicle mounted high resolution multi-spectral 3D data capture platform is presented. As the vehicle traverses a city, it will collect high resolution visual, thermal and 3D scene data and thus facilitate the application of $\mathrm{CV}$ and ML techniques in future large urban studies, such as the automatic categorisation of facade material stocks and evaluation of residential construction conditions and their thermal performances.

\section{DESIGN CONSIDERATIONS}

The core rig data capture components consist of visible-light cameras, radiometric thermal cameras, LiDAR units and a combined Global Navigation Satellite System (GNSS) and Inertial Measurement Unit (INU). The rig aims to be mounted on the roof rack of a vehicle and will capture the aforementioned data from both sides of the road and simultaneously from all sensors as the vehicle drives along the road.

In developing a high performance and reliable vehicle mounted multi-spectral 3D data capture rig, challenges are mainly derived from the following aspects: optimal sensor placement and FOV design, robust and rigid structural design, reliable and accurate data capture and precise multi-sensor data fusion.

The numerous and conflicting design criteria involved makes the development of a state-of-the-art vehicle mounted data capture rig for 3D urban scene generation a non-trivial task. For example, as all optical sensors are integrated on the system bracket, good rigidity and vibration isolation capability are required to ensure the stable viewing conditions of these sensors, however, the overall weight of the rig should also be kept to a minimum in order to not significantly impact the vehicles driving characteristics.

Due to the possibility of sudden changes in environmental factors (ex. rain), the rig design should aim to have an IP rating of at least IP55.

\subsection{Required Sensor FOVs}

To cover the wanted scene, the field of view (FOV) of each imaging unit should be selected correctly. In this case, the mechanical system should be designed with some methods to make finer alignment of the sensors during the assembling and testing phases. Ideally most data capture units should have some freedom for tilting or rotating. When a unit is aligned, it should be secured tightly with the position of each unit marked so that remounting of the unit can be positioned in exactly the same position.

When the vehicle drives along the road, the FOV of each unit should cover the buildings beside the road, especially the height of buildings. As residential buildings are of primary interest, data capture of two to three storey high buildings is required. The required FOV is different for the near-side buildings and far-side buildings. Capturing the near-side buildings requires a larger FOV for each imaging unit than capturing the far-side buildings.
As factors such as the road width, number of lanes and pedestrian walkways are variable and cannot be controlled, exact minimum FOVs cannot be stated. However, the minimum FOVs for a typical single carriageway with two lanes can be used to get rough idea of the approximate FOVs required. Given that the baseline of a sensor is capable of being tilted, the approximate FOVs to capture a 3 storey building facade can be calculated by

$$
\begin{aligned}
\theta_{N, 3}+\alpha_{N} & =\arctan ((H 3-H) / D N)+\arctan (H / D N) \\
\theta_{F, 3}+\alpha_{F} & =\arctan ((H 3-H) / D F)+\arctan (H / D F)
\end{aligned}
$$

where $\mathrm{H}$ is the height of the sensor; DF is the distance between the sensor and the far-side building; DN is the distance between the sensor and the near-side building; $\mathrm{H} 2$ is the height of a two storey building; $\mathrm{H} 3$ is the height of three storey building; $\theta_{F, 2}$ is the angle of the half-FOV covering a far-side two storey building; $\theta_{F, 3}$ is the angle of the FOV covering the half-FOV covering a far-side three storey building; $\theta_{N, 2}$ is the angle of the half-FOV covering a near-side two storey building; $\theta_{N, 3}$ is the angle of the half-FOV covering a near-side three storey building. This is illustrated in Figure 1.

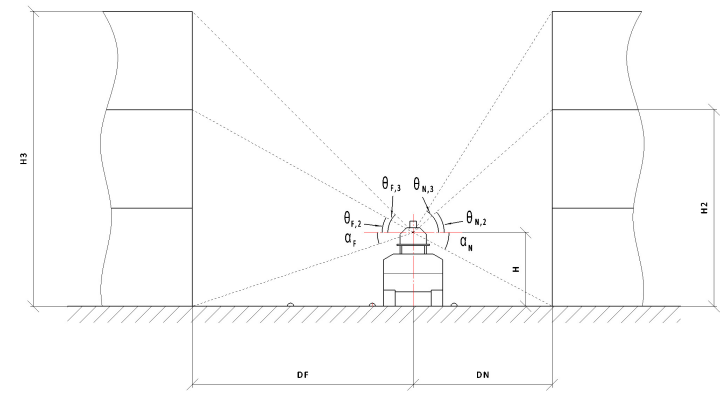

Figure 1: Cross-section diagram of a sensor's potential field of view.

Standardised widths for new UK roads were set in 1993. This set the standardised width of a single lane at $3.65 \mathrm{~m}$ and thus a typical single carriageway with two lanes (i.e. one lane for each direction) being $7.3 \mathrm{~m}$ wide. However, standard residential streets can be as narrow as $5.5 \mathrm{~m}$ depending on predicted traffic levels and number of access points on the road, such as driveways.

Under the assumption that the vehicle mounted sensor is positioned at a height of $2.25 \mathrm{~m}$, the two-lane road width is assumed to $5.5 \mathrm{~m}$, the distance between the road curb and the building is $3 \mathrm{~m}$ and finally the height of each storey is $3 \mathrm{~m}$, then the approximate required FOV is $68^{\circ}$ for a near-side two storey building, $84.3^{\circ}$ for a near-side three storey building, $45.3^{\circ}$ for a far-side two storey building and $61^{\circ}$ for a far-side three storey building.

\section{SENSORS}

The Ladybug5+ visual camera rig $(30 \mathrm{~Hz}$, resolution: $2048 \times 2448$ pixels, FOV: $90 \%$ of full sphere, IP65) is composed of six separate Sony IMX264 CMOS sensors with one on the top pointing upwards and the other five positioned along the sides forming a pentagon. The integrated FOV of a Ladybug $5+$ camera covers a $360^{\circ}$ sphere which meets the required FOV. 
The FLIR A615 LWIR camera $(50 \mathrm{~Hz}$, resolution: $640 \times 480$ pixels, FOV: $45^{\circ} \times 34^{\circ}$, Spectral Range of $7.5-14 \mu \mathrm{m}$, IP30) was chosen as the radiometric thermal cameras to be used on the rig. The FOV of these cameras did not come close to meeting the expected minimum FOV for a near-sided three storey building thus it is necessary to assemble two A615 cameras into a dual thermal camera mount to obtain an almost double vertical FOV. To capture both sides of the rig, a total of four FLIR A615 LWIR cameras are thus required. The FLIR A615 LWIR cameras does not meet the IP rating criteria and thus requires housing to achieve an IP rating as close to or in excess of IP55 if possible. It is beneficial to position the thermal cameras along the same vertical plane as the Ladybug $5+$, however this may have a significant impact on the overall rig design if these sensors' FOV is obstructed by the other sensor.

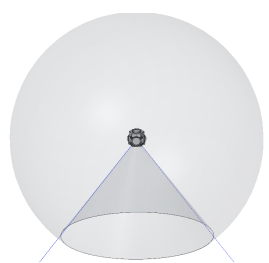

(a) Visual

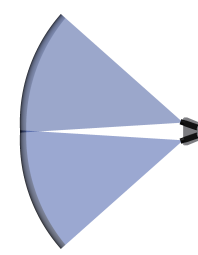

(b) Thermal

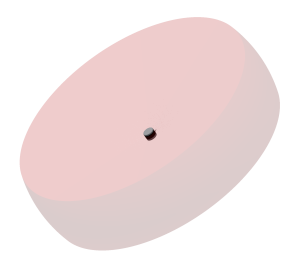

(c) LiDAR
Figure 2: Illustrated Field of Views (FOVs) for: (a) the visiblelight Ladybug5+ camera unit; (b) the duel radiometric thermal A615 camera setup; and (c) an individual VLP-16 LiDAR unit.

Four Velodyne VLP-16 LiDAR units $(20 \mathrm{~Hz}, 16$ laser beams, range: $100 \mathrm{~m}$, FOV: $360^{\circ} \times 30^{\circ}$, IP67) will be used in this rig to have optimal coverage of the built environment. Each Velodyne LiDAR offers a FOV of $+/-15^{\circ}$ ( $360^{\circ}$ scanning) as shown by Figure 2c. To optimally cover the buildings beside the road, the LiDAR units will all be tilted $45^{\circ}$ and each mounted on the rig at $90^{\circ}$ spacings from each other. This positional arrangement is shown in Figure 3a. By this arrangement, each LiDAR unit's FOV captures data from both sides of the rig.

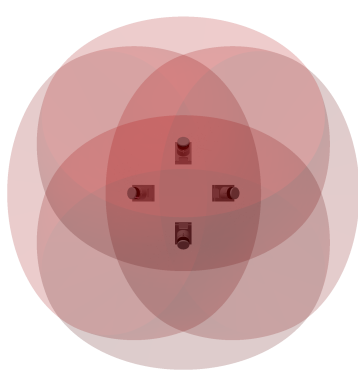

(a) LiDAR FOV setup

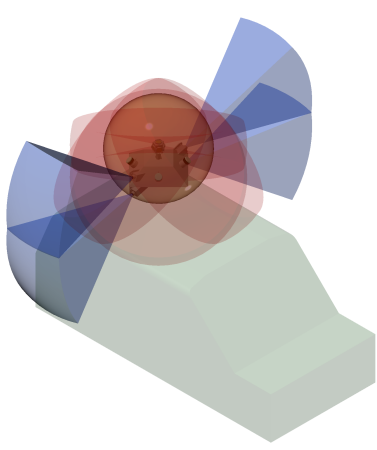

(b) Combined sensor setup
Figure 3: Illustrated Field of Views (FOVs) for: (a) the four VLP-16 LiDAR unit setup; and (b) the overall combined approximate sensor positions.
The OXTS xNAV is a duel antenna GNSS/IMU localisation unit which combines GPS, GLONASS, an IMU and RTK correction signals to produce open sky localisation errors as low as $2 \mathrm{~cm}$.

Based on the aforementioned individual sensors' FOV setups, Figure $3 \mathrm{~b}$ shows a combined approximate FOV and sensor position layout. The Ladybug5+ is positioned at the top of the rig with the A615 thermal cameras positioned each side of the rig. Finally, the LiDAR units are positioned squarely around the outside of the rig. In the structural design of the rig, all sensor positions and mounting fixtures should be carefully considered to gain the optimal FOV of the built environment whilst avoiding obstruction from other sensors and components of the rig.

\section{STRUCTURAL DESIGN}

\subsection{Initial Concept}

Figure 4 presents a rendering of the rig's initial concept design. The mechanical structure of the rig can be decomposed into an internal mounting bracket (i.e. Figure 4a) and external rig covers (i.e. Figure $4 \mathrm{~b}$ ). The rig covers are cut $3 \mathrm{~mm}$ aluminium sheets that are designed to protect specific sensors, cables and sockets from environmental factors such as water, dust and insects. The highlighted blue components shown in Figure $4 \mathrm{~b}$ are fabricated mouldings designed to reduce the wind resistance when driving at a high speed. The rig is intended to be mounted on the roof of a vehicle via a rack connection.

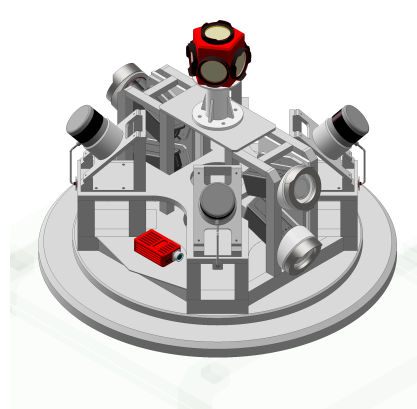

(a) Rig without covers

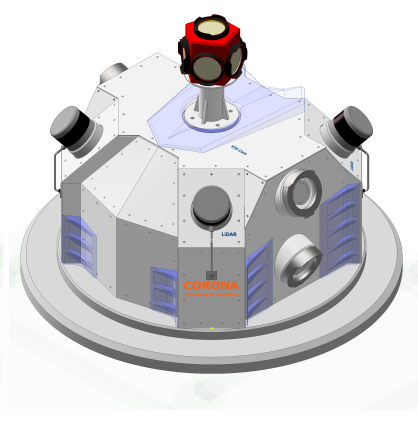

(b) Rig with covers
Figure 4: Initial concept design of a vehicle mounted integrated sensing platform.

The internal bracket shown in Figure 4a is designed to support the imaging units and GNSS/IMU module. The bracket can be divided to three levels with the Ladybug5+ mounted on the top layer, the four LiDAR units mounted on the middle layer and the GNSS/IMU mounted on the bottom layer. The thermal camera units will be inserted into the bracket on the left and right side. All cables can be gathered to the centre hole and lead down into the vehicle carriage. The internal bracket would be assembled from aluminium plates, square tubes and corner brackets that would connect the plates and tubes together.

The Ladybug $5+$ is held by a $150 \mathrm{~mm}$ high post that can be made from a round tube and two flanges. Four ribs would be welded between the tube and lower flange to enforce the rigidity of the 
post. Cables from the Ladybug $5+$ can be led to the rig through the centre of the tube.

As shown in Figure 4, the four LiDARs are mounted on the rig in a square pattern. A hollow round cylinder is used to fix each LiDAR onto the internal bracket while also lifting the LiDAR unit $50 \mathrm{~mm}$ to avoid obstruction of the LiDARs' FOV by the Ladybug5+. The LiDAR units would be mounted on the bracket at $45^{\circ}$ deviating from the middle plane of the van.

While most of the sensor units are sufficiently IP rated, the FLIR A615 cameras require environmental protection during operation. The rig covers provide this protection to the internally housed A615 cameras, and as shown in Figure 5a, IR windows are mounted onto the side covers to allow radiometric thermal data capture from each of these cameras. The IR windows should be tilted at least $5^{\circ}$ from the optical axis of the A615 cameras to reduce the narcissus effect.

Two FLIR A615 cameras would be mounted onto a metal plate to assemble a duel camera unit for each side of the sensing platform. In practice, machining and assembling errors may alter the integrated FOV of this unit, thus aside from adding some slight flexibility to the resulting sensor FOV design, it might also be necessary to adjust the angle of each A615 camera to compensate for these types of errors. Figure $5 \mathrm{~b}$ shows the mechanical design to assemble and adjust thermal camera unit. A short shaft, screwed on the small plate, is inserted into a trapezoidal plate to allow for the rotation of each A615 camera. Two pairs of arc slots are cut on the trapezoidal plate which are centric to each shaft hole. Each A615 camera could rotate around the shaft centre for at least $+/-5^{\circ}$. The whole thermal camera unit would be fixed to the side of the internal bracket.

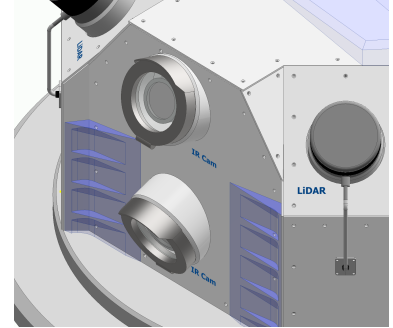

(a) Infrared windows

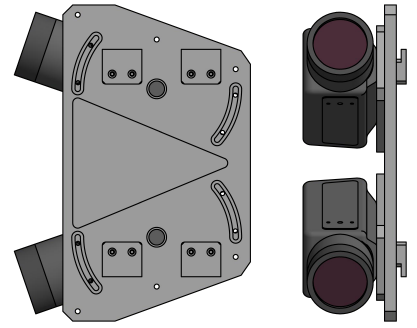

(b) Duel thermal camera unit
Figure 5: Duel FLIR A615 radiometric thermal camera setup and infrared windows.

The GNSS/IMU xNAV unit would be fixed to the base of the rig and connected to two GNSS antenna units. These antenna units are required to be mounted on the vehicle roof and placed 2 meters apart from each other.

\subsection{Evaluation}

From a structural perspective the concept design was assessed to be over engineered for the given task. Some internal bracket components proposed in aluminium were of excessive thickness for the loads they were required to withstand and consequently would have weighed significantly more than necessary. Although rigidity and relative position between components is important, the required design does not appear to need to be as robust as the proposed design. If the rig were to be produced in its current form, the structure would be required to be mostly made out of carbon fibre or a similar composite structure to reduce weight. As while many vehicles could still support the concept rig's significant weight via roof rack mounting points, for ease of rig assembly and removal, vehicle energy efficiency and vehicle handling characteristics, it is important to reduce weight where possible.

The rig covers were also assessed to be unsuitable. Not only were there many odd shapes and changes in the structure's orientation, some aluminium plates were not properly supported from the internal bracket. The rig covers were found to only allow limited access to the internals of the rig and thus the A615 thermal cameras were not easily accessible for FOV adjustment. In fact, any equipment would be difficult to replace if the structure was welded and sealed together. Furthermore, with so many joins it would have been difficult to make the rig water tight as per the intended IP rating. As the majority of individual sensors were sufficiently IP rated as standard, a sizeable rig cover may not actually be required.

Rather than trying to combine all their housings into one, individual housings for sensors could be constructed. This could potentially simplify the structure, whilst also having the benefit of easier access to sensors for adjustment. Similarly, to simplify the overall structure and significantly reduce the size and weight of the rig, the housing for the rig could have been made from a composite structure of one or two large pieces instead of multiple aluminium or steel panels.

Finally, depending on the chosen vehicle, vibrational forces such as drivetrain vibration could be a significant issue to this proposed design. Notably, the A615 radiometric thermal cameras have a recommended $2 \mathrm{G}$ maximum vibration limit and excessive vibration could either cause data reading issues or damage to the unit.

\subsection{Revised Concept}

Presented in Figure 6, the revised concept was designed to maintain the existing spatial relationship between the different sensors as per the initial concept design, whilst overcoming some of the negative aspects that it presented and were discussed in the prior section. The revised concept is based upon an X-shaped structure that allows holding of the central post for the Ladybug5+, transferring the load directly to the mounting points at each corner. The VLP-16 LiDAR units are positioned on the arms of the X-shaped structure.

The main components of the structure can be laser cut in $6 \mathrm{~mm}$ thick sheet aluminium. The panels can be selectively perforated to achieve significant weight reduction. These aluminium panels could slot together and be fillet welded at their intersections. The horizontal shelves triangulate this arrangement to produce good stiffness properties. Through the use of smart profile design for the laser cut parts, it would be possible to assemble and fasten the rig together for welding without requiring any jigs, thus improving production speed.

The rig would sit on a fabricated tubular frame that elevates it up to the required height for all sensor FOVs to be unobstructed by the vehicle body. Rubber anti-vibration mounts were used to mount the rig to the tubular frame. As shown in Figure 7, the tubular frame would be braced horizontally to aid stiffness and is arranged to bear the load back down to the roof bar mounting positions on the vehicle. 


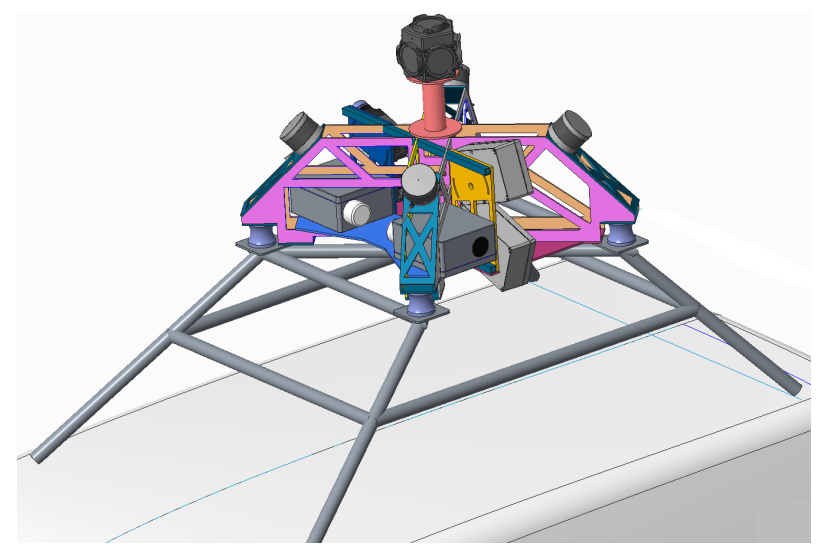

Figure 6: Revised concept design of a vehicle mounted integrated sensing platform.

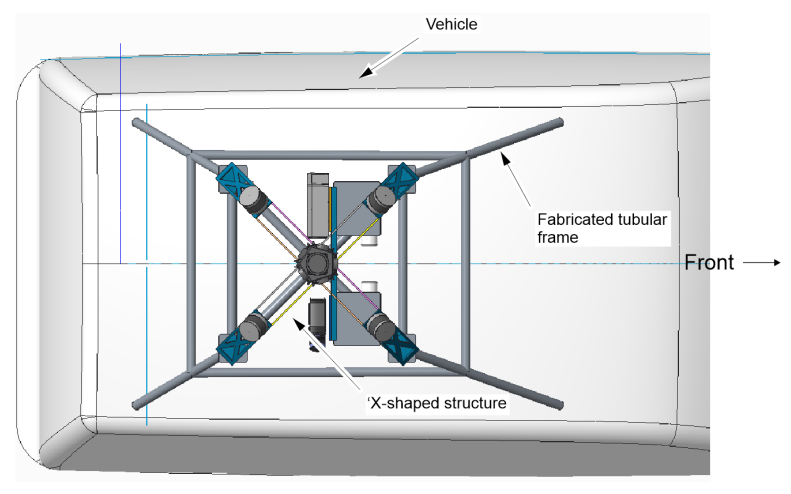

Figure 7: Top-down view of the revised concept design.

Due to the rigid design, it was thought that any sensor addition at a later design or manufacturing stage would result in significant difficulties, thus it was decided at this concept design stage to incorporate a horizontal shelf each side of the rig to flexibly hold additional sensors. The initial sensors intended to be housed on these horizontal shelves are two Specim FX10e hyperspectral cameras. The Specim FX10e hyperspectral camera $(330 \mathrm{~Hz}$, spectral range 400-1000 nm, spectral bands: 224, spatial sampling: 1024 pixels, FOV: $38^{\circ}$, IP30) works in a line-scan mode in the visible and near-infrared (VNIR) area. Like the A615 thermal camera, the Specim FX10e hyperspectral camera does not meet the IP rating criteria and thus would require housing to achieve a sufficient IP rating.

The A615 thermal cameras are mounted on a vertical panel which sits transversely on the rig. Just like the initial concept design, adjustment of the thermal cameras is accomplished via pivoting the about a central mount and using circumferential slots to clamp at different angular positions.

There was no attempt to make the whole structure IP rated. Instead, any sensor that did not have a sufficient IP rating (i.e. the two FX10e hyperspectral cameras and four A615 thermal cameras) would be housed in their own appropriately IP rated enclosure.
To improve the aerodynamic performance of the rig, the forward and rearward spaces subtended by the two arms of the ' $X$ ' could be filled in with two cowlings, as shown in Figure 8. To keep weight down, these could be a glass fibre reinforced components. One drawback of this design's reduced size and requirement to house the thermal cameras in there own IP rated enclosures is that the enclosures would extend beyond the envelope of the rig and therefore reduce aerodynamic performance. Not including the IP rated boxes, sensors, cowlings, fixings and cables, the main structure of this rig design would weigh approximately $30 \mathrm{~kg}$.

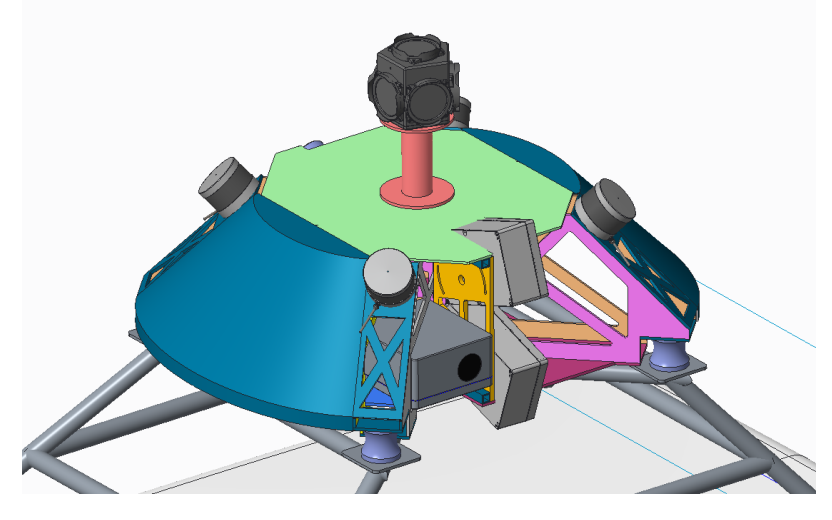

Figure 8: Revised concept design with cowlings.

\section{CONCLUSIONS}

In this paper, a revised concept design for a Vehicle Mounted High Resolution Multi-Spectral 3D Scanner was presented. For this design, the requirements of the rig as a whole, each individual piece of equipment being used, the structural assembly and component materials, the data the rig was required to gather and the environmental/usage conditions when mounted on the roof of a vehicle were all considered.

The main conclusion from reviewing the initial design was that it was over engineered and the weight should be reduced considerably. While the revised concept design improved on many of the negative aspects of the initial concept design, not all negative aspects were able to be resolved. This is in part due to the numerous and conflicting design criteria involved in the design. In particular, due to the compactness of both designs and the interrelationship of different components, accommodate future modifications would likely not be an easy task.

In ongoing and future work, the design of a High Resolution Multi-Spectral 3D Scanner will be further explored and refined before ultimately being manufactured. This would then facilitate the application of CV and ML techniques in future large urban studies.

\section{ACKNOWLEDGMENTS}

This research was supported by the EPSRC Grants; City Observatory Research Platform for Innovation and Analytics [EP/R013411/1] and UK Collaboratorium for Research in Infrastructure \& Cities [EP/P016782/1]. 


\section{REFERENCES}

[1] Mario Ivan Alba, Luigi Barazzetti, Marco Scaioni, Elisabetta Rosina, and Mattia Previtali. 2011. Mapping infrared data on terrestrial laser scanning 3D models of buildings. Remote Sensing 3, 9 (2011), 1847-1870

[2] A. John Arnfield. 2003. Two decades of urban climate research: a review of turbulence, exchanges of energy and water, and the urban heat island. International Journal of Climatology: a fournal of the Royal Meteorological Society 23, 1 (2003), $1-26$.

[3] C. Brenner, N. Haala, and D. Fritsch. 2001. Towards fully automated 3D city model generation. Automatic Extraction of Man-Made Objects from Aerial and Space Images (III) (2001), 47-57.

[4] Alberto Broggi, Paolo Grisleri, and Paolo Zani. 2013. Sensors technologies for intelligent vehicles perception systems: A comparison between vision and 3DLIDAR. In 16th International IEEE Conference on Intelligent Transportation Systems (ITSC 2013). IEEE, 887-892.

[5] Federico Caprotti and Joanna Romanowicz. 2013. Thermal eco-cities: Green building and urban thermal metabolism. International fournal of Urban and Regional Research 37, 6 (2013), 1949-1967.

[6] Shaoqing Chen and Bin Chen. 2015. Urban energy consumption: different insights from energy flow analysis, input-output analysis and ecological network analysis. Applied Energy 138 (2015), 99-107.

[7] Matthew Fox, David Coley, Steve Goodhew, and Pieter De Wilde. 2015. Timelapse thermography for building defect detection. Energy and Buildings 92 (2015) 95-106.

[8] Niki Gaitani, Ingunn Burud, Thomas Thiis, and Mat Santamouris. 2017. Highresolution spectral mapping of urban thermal properties with Unmanned Aerial Vehicles. Building and Environment 121 (2017), 215-224.

[9] Edmundo Guerra, Rodrigo Munguia, and Antoni Grau. 2018. UAV visual and laser sensors fusion for detection and positioning in industrial applications. Sensors
18,7 (2018), 2071

[10] Jeffrey R Kenworthy. 2006. The eco-city: ten key transport and planning dimensions for sustainable city development. Environment and Urbanization 18, 1 (April 2006), 67-85. https://doi.org/10.1177/0956247806063947

[11] Loic Landrieu and Martin Simonovsky. 2018. Large-scale point cloud semantic segmentation with superpoint graphs. In Proceedings of the IEEE Conference on Computer Vision and Pattern Recognition. 4558-4567.

[12] Jesus Morales, Victoria Plaza-Leiva, Anthony Mandow, Jose Gomez-Ruiz, Javier Seron, and Alfonso Garcia-Cerezo. 2018. Analysis of 3D scan measurement distribution with application to a multi-beam lidar on a rotating platform. Sensors 18, 2 (2018), 395.

[13] Salem Morsy, Ahmed Shaker, and Ahmed El-Rabbany. 2017. Multispectral LiDAR data for land cover classification of urban areas. Sensors 17, 5 (2017), 958.

[14] Stephan Nebiker, Susanne Bleisch, and Martin Christen. 2010. Rich point clouds in virtual globes - A new paradigm in city modeling? Computers, Environment and Urban Systems 34, 6 (2010), 508-517.

[15] Daniel O’Donohue, Steven Mills, Simon Kingham, Phil Bartie, and David Park. 2008. Combined thermal-LIDAR imagery for urban mapping. In 2008 23rd International Conference Image and Vision Computing New Zealand. IEEE, 1-6.

[16] Sang-Il Oh and Hang-Bong Kang. 2017. Object detection and classification by decision-level fusion for intelligent vehicle systems. Sensors 17, 1 (2017), 207.

[17] Mark Roseland. 1997. Dimensions of the eco-city. Cities 14, 4 (1997), 197-202.

[18] Michiel Vlaminck, Hiep Luong, Werner Goeman, and Wilfried Philips. 2016. 3D scene reconstruction using Omnidirectional vision and LiDAR: A hybrid approach. Sensors 16, 11 (2016), 1923.

[19] Chao Wang, Yong K. Cho, and Mengmeng Gai. 2012. As-is 3D thermal modeling for existing building envelopes using a hybrid LIDAR system. Fournal of Computing in Civil Engineering 27, 6 (2012), 645-656. 